\title{
Design on the Absorbing Composites Using the Frequency-Advantage to Layer-stacked Rule
}

\author{
Yong-Gang XU ${ }^{1, a,{ }^{*}}$, Li-Ming YUAN ${ }^{1, a}$, Fang-Yuan CHEN ${ }^{1, a}$ and Qi-Lin WU ${ }^{2, d}$ \\ ${ }^{1}$ Science and Technology on Electromagnetic Scattering Laboratory, Shanghai, 200438, China \\ ${ }^{2}$ Key Laboratory of High Performance Fibers \& Products, Ministry of Education, Donghua University, \\ Shanghai, P.R. China, \\ axuyonggang221@163.com, ${ }^{\mathrm{b}}$ Iming_y@163.com, ${ }^{\mathrm{c} f a n g y u a n s c u @ g m a i l . c o m},{ }^{\mathrm{d}}$ anicemoon@sina.com \\ ${ }^{*}$ Corresponding author: Yonggang $\mathrm{Xu}$
}

Keywords: layer-stacked; absorbing materials; FeSi; CIPs; reflection loss; shielding effectiveness.

\begin{abstract}
A frequency-advantage to layer-stacked rule was proposed to design the wide band absorbing materials. The two stacked layer was constructed by the silicone rubber composites added the FeSi alloys and the carbonyl iron particles (CIPs). The layer permittivity and permeability were measured using a vector network analyzer, and then the reflection loss (RL) and the shielding effectiveness (SE) of the two type layer were calculated respectively. The results indicated that composites added FeSi had the higher permittivity and permeability at 1-4 GHz while the composites added CIPs had the better absorbing property at $8-18 \mathrm{GHz}$ due to the characteristics of the two absorbents. Then using a genetic algorithm method on optimizing the wide frequency, the absorbing property of the two advantage frequency could be kept. The absorbing band was widened to 2.3-18 $\mathrm{GHz}$ as RL was lower than $-8 \mathrm{~dB}$ with the total thickness less than $3 \mathrm{~mm}$. The frequency-selected to layer-stacked rule was an effective method on designing the absorbers with the wide frequency absorbing band.
\end{abstract}

\section{Introduction}

Absorbing the microwave is one of the effective ways to prevent the microwave hazards on the human health and the electrical equipment. The variable absorbents, such as the Fe alloys, the carbonous material, the nano-absorbent, the conducting polymer micro particle, the chiral material, or the frequency selected surface, et al, were gradually studied in depth. However, the absorbing materials fixed on the electronic devices was still limited, their characteristics including the wide absorbing band, the large thickness, the density and the high absorption were not met [1]. In order to achieve those characteristics, recently the metal alloys were used widely in the absorber due to the high magnetic permeability in $\mathrm{GHz}$, because the matching characteristic and attenuation characteristic could be satisfied much more easily than other type absorbent [2]. The frequency range of 1-18 GHz was commonly used for the military and civil equipment. It was very difficult to select a single absorbent to achieve the wide absorbing band as the absorber thickness was limited to the millimeter size. For example, the flaky Fe alloys (FeSi, FeSiAl, FeNi, et al) have been extensively used as the soft magnetic metal material due to their large values of saturation magnetization and large Snoek's limit at frequency 1-4 GHz [3]. The results showed that RL of the composites filled with flaky FeSiAl was $-7.5 \mathrm{~dB}$ at $1 \mathrm{GHz}$ [4], and these particles were suitable absorbents used in 1-4 $\mathrm{GHz}$ [5]. The flaky CIPs alloys were oftenly used in the absorbing plate or structure due to their excellent absorbing property in frequency of 8-18 GHz. Although the two $\mathrm{Fe}$ alloys were effective in the separated band, how to take advantage of the two typical particles could be valuable on designing the excellent absorbing composite, and it might break through the limitation on the wide band absorbing materials.

The objective of this work is to design the wide band microwave absorbing materials by using the two type absorbents. The two absorbents were added to the matrix respectively. Then the absorbent 
based on the frequency characteristic was analyzed using the transmission/reflection theory. Finally, the RL of the composites was calculated and measured in frequency range of 1-18 GHz.

\section{Materials and Experiment}

\section{Materials Preparation and Measurement}

Flaky FeSi and CIPs particles were prepared by the mechanical milling process, in which the $\mathrm{ZrO} 2 \mathrm{jar}$ and ball were used and the n-hexane and stearic acid was added as the dispersant. The raw commercial particles of the spherical shape were supplied by Changsha Hualiu Power Co. Ltd and Shanxi Xinghua Power Co. Ltd, China. The morphology of the two particles can be shown in Fig.1. The average diameter of two milled particles was $100 \mu \mathrm{m}$ and $5 \mu \mathrm{m}$ respectively. Then the silicone rubber was used as the matrix to fabricate the testing sample, and the mixing process was done as the two particles were added to the matrix.
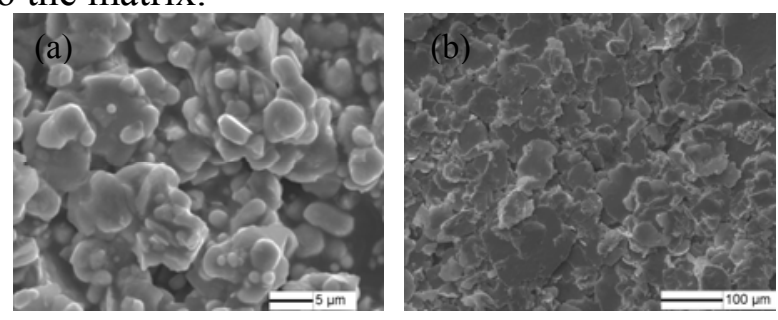

Fig.1 SEM images of the two particles, (a) flaky CIPs and (b) flaky FeSi.

The particle morphology was observed by the scanning electron microscopy (SEM CamScan CS3400). The effective complex permittivity and permeability of the absorbers were measured on a vector network analyzer by the transmission method in 1-18 GHz. The testing samples for EM parameters measurement were shaped to a toroidal shape with an outer diameter $7.0 \mathrm{~mm}$, inner diameter $3.04 \mathrm{~mm}$ and thickness $2 \mathrm{~mm}$. Then the scattering parameters $S_{11}$ and $S_{12}$ could be measured, and the permittivity $(\varepsilon r)$ and the permeability $\left(\mu_{\mathrm{r}}\right)$ could be deduced using the Nicolson-Ross-Weir algorithm.

\section{Frequency-selected to Layer-stacked Rule}

The frequency- selected to layer-stacked rule was proposed based on the transmission/reflection characteristics of the absorbers. The frequency band 1-18 GHz was separated into two parts, $1-4 \mathrm{GHz}$ and 4-18 GHz, and the absorbing composite of two layer structure was selected according to the two frequency ranges. The detailed microwave transmission and reflection diagram is showed in Fig. 2.

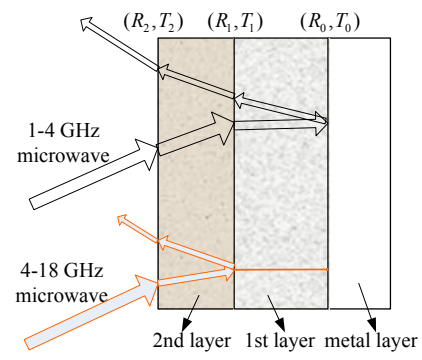

Fig.2 The frequency-advantage design schematic diagram

As the microwave of the frequency $1-4 \mathrm{GHz}$ transmitted on the layer surface, the first layer materials had the good transmission efficiency, the microwave transmitted to the surface of the second layer, the microwave could be attenuated in the second layer, and the reflected microwave energy was small. Then the attenuated microwave transmitted through the first layer, as a result, the microwave was attenuated. While as the microwave with the frequency $4-18 \mathrm{GHz}$ transmitted to the first layer surface, the microwave could be transmitted into the layer and could be directly attenuated in the first layer, the attenuated microwave then transmitted to the second layer surface, the 
microwave could be mostly reflected due to the better shielding property of the second layer, the reflected microwave could be continually attenuated in the first layer. So, based on the characteristic of the two type absorbing materials, the microwave with the total frequency $1-18 \mathrm{GHz}$ could be attenuated. The integrated high-frequency and the low-frequency absorbing layer were effective in order to design the wide band absorbing materials.

The absorbing property of the layer-stacked absorber was calculated based on the permittivity, the permeability and the thickness of the layers. For the multi-layer absorbing plate, the input impedance of the $n$th layer $\left(Z_{i n n}\right)$ is expressed as follows,

$$
\begin{aligned}
Z_{\text {inn }} & =Z_{n} \frac{Z_{\text {inn-1}}+Z_{n} \tanh \left(\gamma_{n} d_{n}\right)}{Z_{n}+Z_{\text {inn- } 1} \tanh \left(\gamma_{n} d_{n}\right)} \\
Z_{\text {in } 1} & =Z_{1} \operatorname{th}\left(\gamma_{1} d_{1}\right)
\end{aligned}
$$

where, of the $n$th layer, $Z_{n}=\sqrt{\mu_{n} / \varepsilon_{n}}$ is the characteristic impedance, $\varepsilon_{n}$ and $\mu_{n}$ were the permittivity and permeability, $\gamma_{n}=i 2 \pi f \sqrt{\varepsilon_{n} \mu_{n}} / C$ is the propagation constant, $d_{n}$ is the thickness, $C$ is the microwave velocity. Then $R L$ of the absorber layer could be derived as follows.

$$
R L=20 \lg \left|\left(Z_{\text {inn }}-Z_{0}\right) /\left(Z_{\text {inn }}+Z_{0}\right)\right|
$$

\section{Results and Discussion}

\section{The Electromagnetic Property of the Two Type Absorbers}
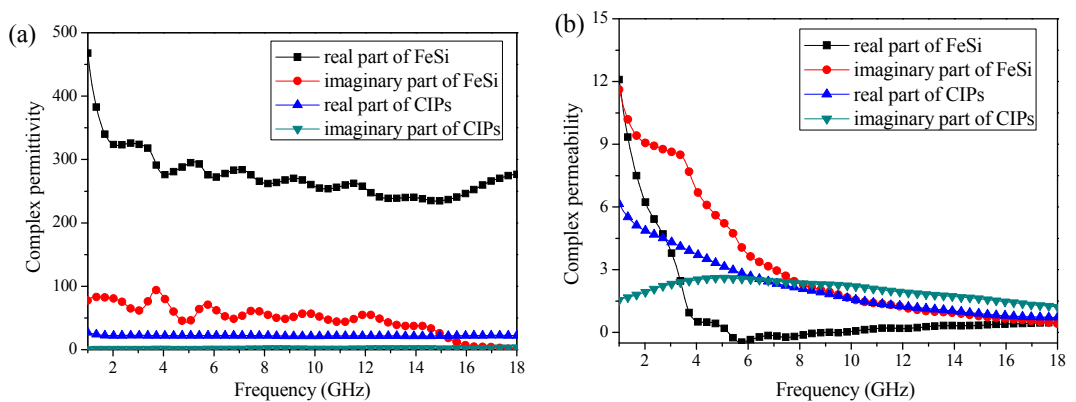

Fig. 3 EM parameters of composites in $1-18 \mathrm{GHz}$, (a) the permittivity, (b) the permeability.

Fig. 3 shows the complex permittivity and permeability of the composites added CIPs or FeSi in the frequency range of 1-18 GHz. It could be clearly obtained that the composite added the FeSi particles had the much larger real part of permittivity $\left(\varepsilon^{\prime}\right)$ and imaginary part of permittivity $\left(\varepsilon^{\prime \prime}\right)$ than that added the CIPs. As the frequency was of 1-4 GHz, the maximum value of $\varepsilon^{\prime}$ was 467.5 as the frequency increased, and it decreased steeply, while $\varepsilon^{\prime \prime}$ did not change obviously, the average value was about 80 . As the frequency was of $4-18 \mathrm{GHz}, \varepsilon^{\prime}$ decreased smoothly and kept fluctuated, the average value could be 267.6, and $\varepsilon^{\prime \prime}$ also kept fluctuated and decreased. Of the CIPs composite $\varepsilon^{\prime}$ and $\varepsilon^{\prime \prime}$ nearly kept constant, the value was about 22.2 and 2.01 respectively. The real part of permeability $\left(\mu^{\prime}\right)$ of the two samples decreased steeply in 1-4 GHz, the maximum value could be obtained 12.09 and 6.15 respectively at $1 \mathrm{GHz}$. The imaginary part of the permeability ( $\left.\mu^{\prime \prime}\right)$ of the FeSi composite decreased as the frequency increased, and $\mu^{\prime \prime}$ of the CIPs increased first and then decreased respectively.

Fig. 4 shows the RL and SE of the two composites in 1-18 GHz with thickness $0.5 \mathrm{~mm}$. The RL defined as the ratio of the reflected power to the incident power with the sample backed by the metal surface and the shielding effectiveness (SE) defined as the ratio of the transmitted power to the incident power through the materials can be represented in the following equations [6]:

$$
\begin{aligned}
& R L(d B)=20 \log \left|\Gamma_{0}\right| \\
& S E(d B)=-20 \log \left|\left(1-\Gamma_{0}^{2}\right) T /\left(1-T^{2} \Gamma_{0}^{2}\right)\right|
\end{aligned}
$$


Where $\Gamma_{0}$ is the reflection coefficient, $\Gamma_{0}=\left(Z_{\text {in }}-1\right) /\left(Z_{\text {in }}+1\right), Z_{\text {in }}$ is the normalized input impedance of the microwave absorbing composites, $Z_{i n}=\sqrt{\mu_{r} / \varepsilon_{r}} \tanh \left(j 2 \pi d \sqrt{\mu_{r} \cdot \varepsilon_{r}} / \lambda\right), T$ is the transmission coefficient, $T=\exp \left(-j 2 \pi d \sqrt{\mu_{r} \cdot \varepsilon_{r}} / \lambda\right)$, and $\varepsilon_{r}$ and $\mu_{r}$ are the complex permittivity and complex permeability of the absorbing composites respectively, $\lambda$ is the wavelength of the microwave, and $d$ is the absorber thickness. Of the FeSi composite, the minimum RL could be obtained at $2.3 \mathrm{GHz}$, and the RL in $1-4 \mathrm{GHz}$ was lower than $-2.5 \mathrm{~dB}$. It could be deduced that as the thickness increased, RL of the FeSi composite could be much lower. While for the CIPs composite, the good absorbing frequency of the absorbing material was about 8-18 GHz, the RL value was lower than $-5 \mathrm{~dB}$. In addition, both the SE values of the two absorbers gradually increased as the frequency increased, and SE of the FeSi composite is much higher than the CIPs absorbing material, it was more than $20 \mathrm{~dB}$ in frequency 6-18 GHz. For the shielding property, SE was determined by the microwave of the reflecting surface and the internal absorption of the absorber. It could be easily observed that the absolute RL was much smaller than SE of the FeSi composite in 4-18 GHz. So the FeSi composite could be used as the reflected layer in $4-18 \mathrm{GHz}$, and as the absorbing layer 1-4 GHz, the CIPs composite could be used as the attenuated coating in 4-18 GHz and as the transmitted coating in 1-4 GHz. The filtering line of the two absorbing materials could be denoted in Fig.4. In another word, the FeSi composites could be used as the advantage absorber at the lower frequency 1-4 GHz, while the CIPs composites could be used as the advantage absorber at the higher frequency 4-18 GHz. The FeSi composite attenuated the lower frequency microwave and reflected the higher frequency microwave, the CIPs composite transmitted the lower frequency microwave and attenuated the higher frequency microwave.
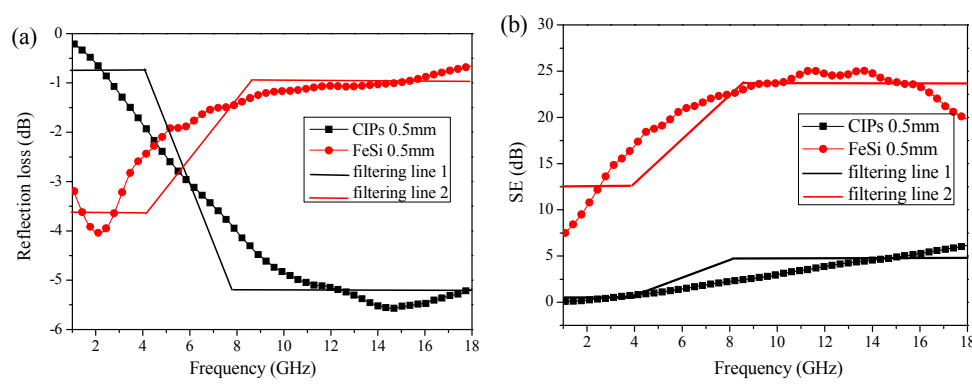

Fig.4 RL and SE of the two kind absorbers with single layer with thickness $0.5 \mathrm{~mm}$, (a) RL, (b) SE

In order to demonstrate the stacking effect of the two type absorber on the electromagnetic microwave, the reflection/transmission coefficient of the two-layer absorber with the two type absorber was analyzed. Fig. 5 shows the four different circumstances of the absorbers, including the single layer added FeSi or CIPs, the two-layer absorber and the two-layer absorber backed by a metal plate. The reflection/transmission coefficients of the four kind circumstances were calculated as shown in Fig.6.

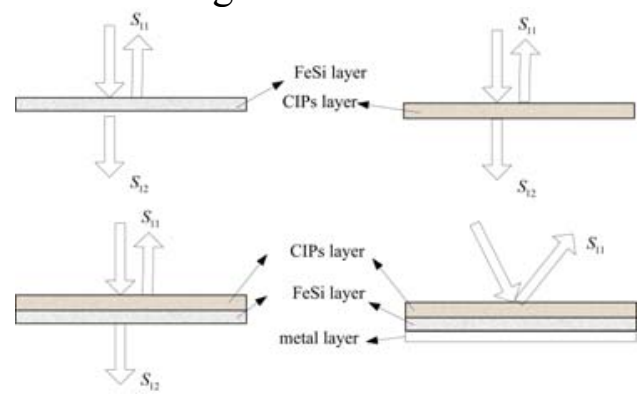

Fig.5 The reflection/transmission property of the two type absorber
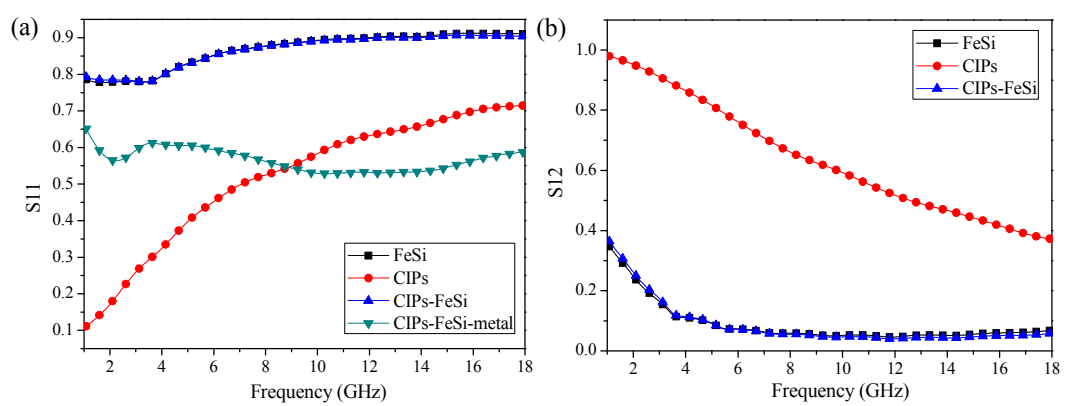

Fig. 6 The reflection/transmission coefficient of the two kind absorbers, (a) $S_{11}$, (b) $S_{12}$

It could be obtained the single FeSi layer had a large $S_{11}$ and large $S_{12}$ in 1-4 GHz, which was consistent with the good absorbing property at 1-4 GHz, and $S_{11}$ was close to 0.9 and $S_{12}$ was close to 
0 in $8-18 \mathrm{GHz}$ which meant that the absorbing property at the frequency band was very weak. While the CIPs layer had the small $S_{11}$ and large $S_{12}$ in 1-4 GHz, $S_{11}$ increased and $S_{12}$ decreased as the frequency increased to $18 \mathrm{GHz}$, which meant that the absorbing property increased. Compared with the two layer absorber with the single FeSi layer, it could be found that $S_{11}$ and $S_{12}$ was very close, and as the metal plate was backed to the two layer absorber, the $S_{11}$ value decreased in the low frequency, as well as the high frequency range. It could be considered that the absorbing in the low frequency range could be kept partly, while the absorbing property in the high frequency could be weak because the $S_{11}$ value was smaller than that of the single CIPs layer absorber.

\section{Enhancement on the Absorbing Property by Optimizing the Stacked Layer}

According to the previous analysis on the two type absorbers, if the high frequency absorber could be optimized, the absorbing property in the high frequency range might be also enhanced. The genetic algorithm was used to optimizing the layer characteristics and the thickness of the two absorbing materials respectively. The RL requirement of the two frequency band could be given, in this work RL we selected was less than $-10 \mathrm{~dB}$ in $8-18 \mathrm{GHz}$, and less than $-4 \mathrm{~dB}$ in 1-4 GHz. So, using an interpolation process on the composite with the random volume content, the selecting composite in the two frequency bands would be optimized respectively. The genetic algorithm could be described as follows, firstly the electromagnetic parameters library was built based on the testing composite added certain volume content absorbent, so the composites filled with variable volume content were derived. Then the electromagnetic parameters and the thickness of each layer were chosen, the chromosome of the genetic algorithm was established, it contains the information including its material number, volume content and thickness. $A i$ and $B_{j}$ were defined to represent the material thickness and the volume content respectively, the bit sequence of layer $n$ is $A_{n} B_{n}$, and the total layer chromosome can be expressed like $A_{1} B_{1} A_{2} B_{2} \ldots A_{n} B_{n}$. In the optimized process, the random population containing 1000 chromosomes was created, then the number of material choice was decoded, the electromagnetic parameters could be used to calculate the RL of each individual, then the select operation, cross operation and mutate operation was done, the next calculation population was created, the previous computation process was done repetitively as the generation number reach to 20 , the optimized result could be obtained. The component of the optimized composite was showed in Table 1. In the optimized composite, the layer number was 3 or 4 . The symbol for the material type Fe-30 denotes the composite added 30\% volume content flaky CIPs, FeSi-30 denotes the composite added $30 \%$ volume content flaky FeSi, the others symbols could be explained similarly.

Table 1 Design on the RL of composite using the optimizing method

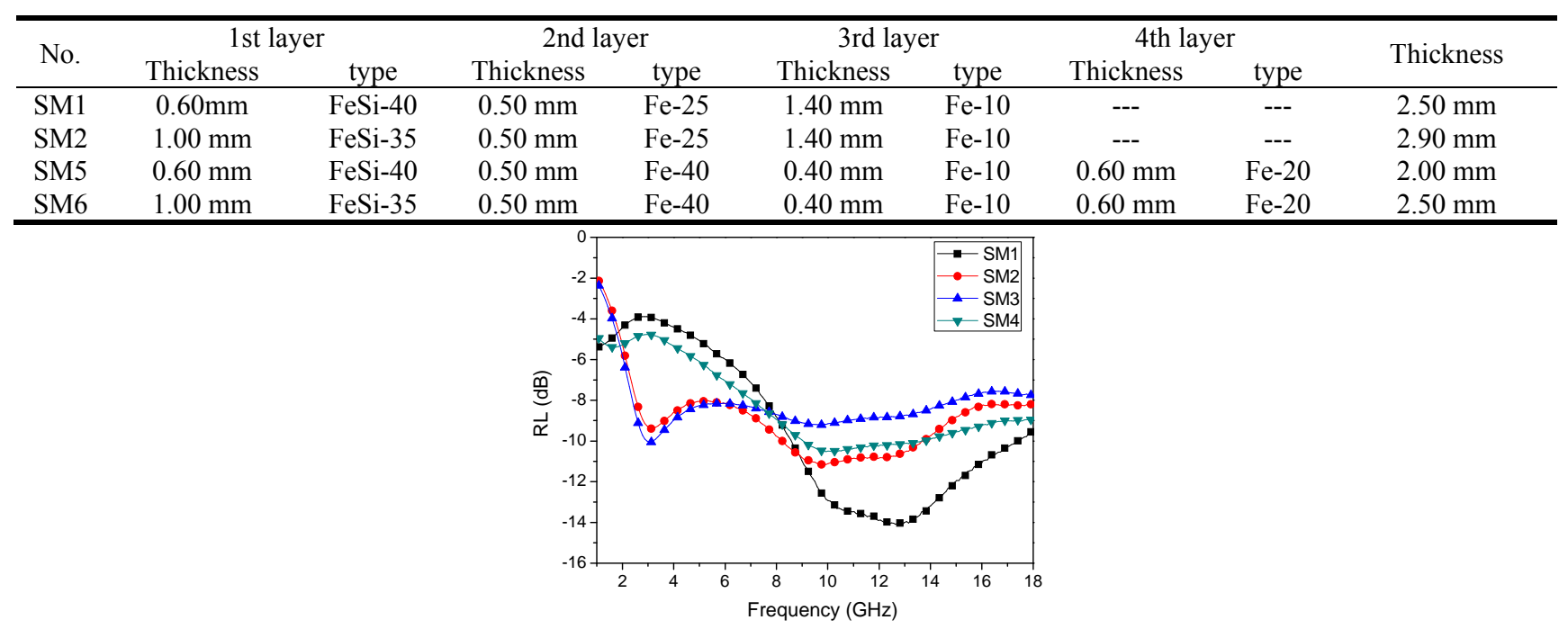

Fig.7 RL of the high frequency absorber of two kind layer in 1-18 GHz, (a) the first kind, (b) the second kind. 
Fig. 7 shows the optimized RL with variable low advantage frequency absorber thickness as a function of the frequency. It could be obtained that as the FeSi layer thickness increased from $0.6 \mathrm{~mm}$ to $1 \mathrm{~mm}$, the RL in 1-4 GHz decreased obviously, but not monotonously, while RL in $8-18 \mathrm{GHz}$ increased but the total value was still less than $-8 \mathrm{~dB}$, it meant that the low frequency absorber had a negative effect on the absorbing property in $8-18 \mathrm{GHz}$, which was consistent with the result of the previous two layer absorbers. Take SM1 and SM5 for example, as the FeSi absorber thickness was $0.6 \mathrm{~mm}$, the RL in $1-4 \mathrm{GHz}$ was $-5.83 \mathrm{~dB} \sim-3.90 \mathrm{~dB}$ and the RL in 8-18 GHz still kept less than $-10 \mathrm{~dB}$. While the $35 \% \mathrm{FeSi}$ absorber thickness was $1 \mathrm{~mm}$, the RL in 1-2 GHz was larger than SM1, and RL in 2-4 GHz decreased steeply, the absorbing frequency with RL less than -8dB could be widened to 2.3-18 GHz, which could be seen an effective method on design the absorber of wide absorbing band.

Oftenly, the good absorbing material should satisfy two important conditions as far as possible, the matching characteristics and the attenuation characteristics. The composite filled with FeSi had the larger dielectric loss tangent and magnetic loss tangent in the frequency range of 1-4 GHz while absorber added the CIPs had the larger loss tangent in 8-18 GHz. Although the two type layer of the absorbing composite was studied only, yet the particle dispersion density and internal multi-layer structure affected the absorbing property of the absorbing materials, so the detailed internal structure of the composite could be considered in the further research.

\section{Summary}

The layer-stacked material could enhance the RL of the composites with thin thicknesses. It was attributed to the characteristics of the FeSi and CIPs composites. The first layer could be used as the transmitted and attenuated layer in the two frequency band, and the second layer could be used as the attenuated and the reflected layer in the two frequency band. The added FeSi layer could improve the absorption in 1-4 GHz and widen the absorbing band with RL less than $-8 \mathrm{~dB}$ in a wide absorbing band.

\section{Acknowledgements}

This work was supported by the National Natural Science Foundation of China (Grant No. 61601299, 61471242), Shanghai Pujiang talents program of the Science and Technology Commission of Shanghai Municipality under grant No. 15PJ14333000.

\section{References}

[1] Micheli D, Apollo C, Pastore R, Marchetti M. X-Band microwave characterization of carbon-based nanocomposite material, absorbing capability comparison and RAS design simulation. Compos. Sci. Technol., 70(2010): 400-9

[2] Baoshan Zhang, Yong Feng, Jie Xiong, Yi Yang, Huaixian Lu. Microwave-absorbing properties of de-aggregated flake-shaped carbonyl-iron particle composites at 2-18 GHz. IEEE Transactions on Magnetics. 42(2006): 1778-1781.

[3] Snoek JL. Dispersion and absorbing in magnetic ferrites at frequencies above one Mc/s. Physica,14(1948): 207-17.

[4] T.D. Zhou, P.H. Zhou, D.F. Liang, L.J. Deng. Structure and electromagnetic characteristics of flaky FeSiAl powders made by melt-quenching, J. Alloys. Compd., 484 (2009) 545-549.

[5] G.K. Tian, X.F. Bi, Fabrication and magnetic properties of Fe-6.5\% Si alloys by magnetron sputtering method, J. Alloys. Compd., 502(2010): 1-4.

[6] Yonggang Xu, Liming Yuan, Xiaobing Wang, Deyuan Zhang. Two-step milling on the carbonyl iron particles and design on the absorption using the genetic algorithm, Journal of Alloys and Compounds, 676(2016) 251-259 